\title{
Intra- and extra-dimensional shifts with constant- and variable-irrelevant dimensions in the rat ${ }^{\prime}$
}

FRANK D. TURRISI, BR YAN E. SHEPP, and PETER D. EIMAS, Brown University, Providence, R. I. 02912

Rats, trained on a $C-I$ problem, exhibited no differences between IDS and EDS, whereas rats trained on a V-I problem did. Furthermore, the C-I problem was learned faster than the V-I problem originally. These data suggest that the $C-I$ condition was not sufficient for the acquisition of a mediator in the present cxperiment.

Two-stage theories of discriminative learning (Sutherland, 1959; Zeaman \& House, 1963; Lovejoy, 1965, 1966) assume that Ss, in learning a discrimination, acquire a mediating response to the relevant stimulus dimension and an approach response to the correct cue of that dimension. These responses are assumed to transfer to subsequent discriminations. The implications of these theories have been tested by experimental comparisons of several transfer or shift paradigms. An adequate test of the theories demands that either positive or negative transfer of the relevant mediator be arranged. Certain paradigms either do not meet such demands or require special assumptions which as yet have not been evaluated. In the Reversal Shift (RVS), for example, positive transfer of the relevant mediator is confounded with negative transfer of the instrumental response, making data from the paradigm difficult to interpret (see Shepp \& Turrisi, 1966, for a more complete treatment of this problem).

The Intradimensional Shift (IDS) and Extradimensional Shift (EDS) paradigms provide valid tests of mediational theories. In the IDS the previously relevant dimension remains relevant during shift, providing positive transfer of the relevant mediator. Negative transfer of the relevant mediator is arranged in EDS since the previously relevant dimension is made irrelevant during shift. The theories predict that IDS will be learned faster than EDS and several experiments have confirmed the prediction (e.g., House \& Zeaman, 1962; Shepp and Eimas, 1964; Dickerson, 1966).

Frequently in the comparisons of shifts, the arrays of stimuli are manipulated arbitrarily or in ways which require untested assumptions. An illustration of this point is the frequent comparison of EDS or Nonreversal Shift (NRS) with an RVS. An EDS arranges negative transfer of the relevant mediator, whereas the NRS arranges zero transfer of the relevant mediator. Although the previously relevant dimension becomes irrelevant during both shifts and new stimuli from the previously relevant dimension replace the training stimuli, the arrays in which the new stimuli are presented differ. In the EDS, the irrelevant stimuli differ within trials, allowing $S$ to respond differentially and providing negative transfer of any mediator which was learned in training. In the NRS, on the other hand, the irrelevant stimuli are the same within trials. Since $S$ cannot respond differentially to the now-irrelevant dimension, no negative transfer is possible. In many experiments no distinction is made between the NRS and the EDS, but the distinction implies the theoretical notion that more than one stimulus from a dimension must be present on a trial before mediating responses to that dimension will occur.

House \& Zeaman (1962) found that retardates learned IDS faster than EDS when trained on a two-dimensional problem with the irrelevant dimension variable within trials but found no difference when trained on a problem in which the stimuli on the irrelevant dimension were constant within and between trials. Although no statistical tests were reported, the Constant-Irrelevant (C-I) groups seemed to have learned the training problem faster than the Variable-Irrelevant (V-I) groups, suggesting that the former, having no effective irrelevant visual dimension, was an easier problem. Unfortunately, strong dimensional preference effects preclude any definitive interpretation of the lack of the EDS-IDS differences in the $C$-I groups. The subgroup which shifted from form to color learned the shift in $\mathbf{4 4 . 7}$ geometric mean errors, whereas the group which shifted from color to form took 2.0. For the IDS, the form-to-form shift was learned in 1.8 geometric mean errors and the color-to-color shift was learned in 11.5. The shift performance seems to have been determined by the dimension which was relevant during the shift rather than by the transfer of a mediator which was learned during the training problem. It is possible that no mediator was learned for the C-I groups, for which the training problem may have been essentially a single-dimensional problem.

The present experiment asks whether a mediating response to a C-I dimension is made and subsequently extinguished or inhibited during training, and whether a multidimensional problem is necessary for the formation of a mediator. If the C-I dimension is responded to in training, there should be no difference between C-I and V-I groups in training but there should be EDS-IDS differences on shift for both groups. If the C-I dimension is not responded to, the C-I group should learn the training problem faster than the V-I group. Furthermore, if a multidimensional problem is necessary for the formation of a mediator, only the V-I groups should exhibit EDS-IDS differences on the shift. Shepp \& Eimas (1964) found that a V-I training procedure yielded EDS-IDS differences on shift. The present experiment will provide data for comparable C-I groups and compare the two conditions. METHOD

Twenty-four male, hooded rats, aged 8-9 months, were used. The apparatus, stimuli, pretraining, and training procedures were the same as those of Shepp \& Eimas (1964).

The experimental procedure differed in one respect from that of Shepp and Eimas: In the present experiment all Ss were trained on a problem in which the irrelevant dimension was constant throughout training. That is, if form were irrelevant, all stimuli would be of the same form on all trials of the training problem, and $S$ would have to learn a discrimination involving, e.g., thickness of stripes superimposed upon the forms. The shift problem was identical to that of Shepp and Eimas. Thus, an attempt was made to replicate the Shepp and Eimas experiment exactly, except for C-I rather than V-I training.

\section{RESULTS}

Figure 1 shows the backward learning curves for original learning and shift. The data of Shepp \& Eimas (1964) represent original learning under the V-I condition and are labelled ED(I) and ID(I) for EDS and IDS, respectively. The data from the present experiment (C-I condition) are labelled ED(II) and ID(II) analogously. A Kruskal-Wallace analysis of variance by ranks, done on the error scores of the four groups in original learning, yielded a significant overall effect $(H=14, d f=3, p<.01)$. A series of Mann-Whitney $U$ tests revealed that although ED(I) did not differ from ID(I) and ED(II) did not differ from ID(II), ED(I) and ID(I) taken together took significantly (Mann-Whitney for large samples, $z=3.4, p<.001$ ) longer to learn than $E D(I I)$ and ID(II) taken together.

Since the C-I problem was originally learned faster than the V-I problem, savings scores representing the differences in mean errors between original learning and transfer were used to compare the transfer effects. A Kruskal-Wallace analysis of variance showed a significant $(H=8.52, \mathrm{df}=3, \mathrm{p}<.05)$ overall effect. Groups ID(I) and ID(II) differed $\left(U=21.5, n_{1}=n_{2}=10, p<.01\right)$, Groups ED(I) and ED(II) did not $\left(U=48, n_{1}=n_{2}=12, p>.10\right)$, nor did Groups ED(II) and ID(II) $\left(\mathrm{U}=51.5, \mathrm{n}_{1}=12, \mathrm{n}_{2}=10, \mathrm{p}>.10\right)$.

\section{DISCUSSION}

The data show that the C-I problem was learned faster than the V-I problem and that Groups ED(II) and ID(II) did not differ on the shift problem. There are several possible interpretations of these data. First, and most parsimonious, is that the C-I group did not learn a mediator during training, but learned in the single-unit fashion proposed by Spence (1936). This interpretation implies that a multidimensional problem, with at least one irrelevant 


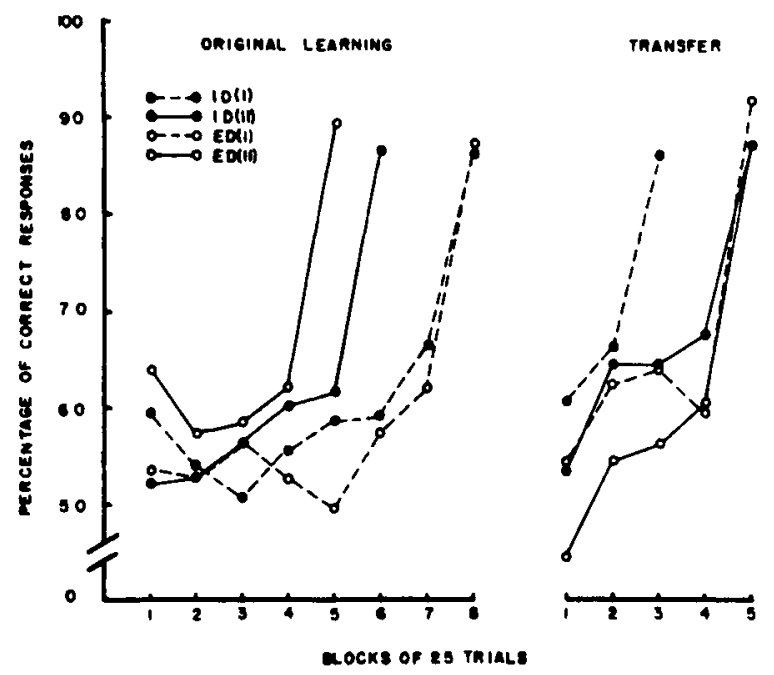

Fi. 1. Backwand keaming curves for percentage correct reaponses as a function of blocks of 25 trials past the point at which the median $S$ was dropped in computing the beckwand curves.

dimension, is necessary to establish a mediator. A second possibility is a novelty effect (House \& Zeaman, 1962). If the C-I dimension is ignored in training, then when it is made variable on the shift it is effectively a novel dimension. On the assumption that the introduction of novel dimensions attracts attention, the IDS would be impaired and the EDS facilitated, since the novel dimension is irrelevant for the former and relevant for the latter. This interpretation loses some force in the present study when it is noted that ED(II) is not facilitated relative to ED(I). A third interpretation is that the C-I Ss learned a mediator to a dimension composed of the compounds of relevant and irrelevant stimuli. There were two such compounds on the training problem and four on the shift problem where the C-I dimension was made V-I. Thus, the shift for all Ss would have been an IDS along a compound dimension from a two-stimulus problem to a four-stimulus problem, resulting in no EDS-IDS differences and slower learning than Group ID(I) which shifted from a two-stimulus problem to another two-stimulus problem (the stimuli along the V-I dimension are not responded to).

Regardless of which theoretical interpretation one chooses to place upon the data, one empirical point emerges. Since the V-I groups showed evidence of mediation, the lack of behavior attributable to mediation in the C-I groups seems to be a function of the stimulus arrangement rather than of a species-specific mediational deficit. Since some paradigms favor mediation whereas other and apparently similar ones do not, it might behoove the investigator to be careful that so-called phyletic or developmental differences in the utilization of mediation are facts of the organism and not artifacts of the paradigm.

\section{REFERENCES}

DICKERSON, D. J. Performance of preschool children on three discrimination shifts. Pyychonomic Science, 1966, 4, 417418.

HOUSE, B. J., ZEAMAN, D. Reveral and nonreveral shifts in discrimination learning of retardates. Journal of Experimental Pyychology, $1962,63,444451$.

LOVEJOY, E. An attention theory of discrimination learning. Journal of Mathematical Psychology, 1965, 2, 342-362.

LOVEJOY, E. Analysis of the overlearning reversal effect. Psychological Review, 1966, 73, 87-103.

SHEPP, B. E., E EIMAS, P. D. Intradimensional and extradimensional shifts in the rat. Joumul of Comparative \& Physiological Prychology, 1964, 57, 357-361.

SHEPP, B. E., TURRISI, F. D. Learning and tnnafer of medinting responses in discriminative learning. In N. R. Ellis (Ed.), Intermational review of research in mental returdetion. Vol. 2 New York: Academic Press, 1966. Pp. 85-121.

SPENCE, $K$. W. The nature of discrimination learning in animals. Psychological Review, 1936, 43, 427-449.

SUTHERIAND, N. S. Stimulus analyzing mechanims. In Proceedtrys of a symposium on the mechenization of thought procesese Vol. 2. London: Her Majesty's Stationery Office, 1959.

NOTE

1. The preparation of this paper was aupported by PHS Grants No. HD-01349-01 and 1-F1-MH-30, 613-01 (MTLH). 